\title{
ON THE TRANSITION FROM CREEPING TO INERTIAL FLOW IN ARRAYS OF CYLINDERS
}

\author{
K. Yazdchi, S. Srivastava and S. Luding \\ Multi Scale Mechanics, University of Twente, CTW, TS, P.O. Box 217, 7500 AE \\ Enschede, Netherlands
}

\begin{abstract}
Many important natural processes involving flow through porous media are characterized by large filtration velocity. Therefore, it is important to know when the transition from viscous to the inertial flow regime actually occurs in order to obtain accurate models for these processes. In this paper, a detailed computational study of laminar and inertial, incompressible, Newtonian fluid flow across an array of cylinders is presented. Due to the non-linear contribution of inertia to the transport of momentum at the pore scale, we observe a typical departure from Darcy's law at sufficiently high Reynolds number (Re). Our numerical results show that the weak inertia correction to Darcy's law is not a square or a cubic term in velocity, as it is in the Forchheimer equation. Best fitted functions for the macroscopic properties of porous media in terms of microstructure and porosity are derived and comparisons are made to the Ergun and Forchheimer relations to examine their relevance in the given porosity and Re range. The results from this study can be used for verification and validation of more advanced models for particle fluid interaction and for the coupling of the discrete element method (DEM) with finite element method (FEM).
\end{abstract}

\section{INTRODUCTION}

The flow of an incompressible fluid through an isotropic porous medium is a physical phenomenon which commonly occurs in ground-water flows and oil reservoirs. Most porous media are granular, but some are composed of very long particles/cylinders and therefore may be described as fibrous media. Common examples of fibrous media include industrial filters, biological tissues and certain polymer membranes. In contrast to these static particle/fiber matrix systems, detailed calculations of heavily loaded, fluid with mobile particle flows are also of interest. Solution methods are mainly based on twofluids (TF) or discrete particle method (DPM) [1, 2]. However, these methods require the knowledge of constitutive closure laws which describe the momentum exchange between the fluid and the particles. Such a correlation is dependent on many parameters of the system, such as the Reynolds number, the solids volume fraction or porosity and the microstructure of the pore domain.

For the case of creeping flow i.e., at near zero Reynolds number $(\operatorname{Re}<<1)$, the pressure gradient and the flow rate have a linear relation, known as Darcy's law:

$-\nabla p=\frac{\mu}{k} U$

where $\nabla p, \mu, U$ and $k$ are pressure gradient, viscosity, superficial velocity and permeability of the porous media, respectively. On the contrary, at small but nonzero Reynolds numbers $(\operatorname{Re} \approx 1)$, the pressure gradient is a nonlinear function of the flow rate as experimentation shown by Forchheimer [3]. He indicated that there exists a quadratic term of flow rate when the Reynolds number is sufficiently high and corrected the Darcy equation into the Forchheimer equation:

$-\nabla p=\frac{\mu}{k} U+\beta \rho U^{2}$ 
where $\rho$ is the density of the fluid and the constant $\beta$, is referred to as the non-Darcy coefficient and, like the permeability, is an empirical value that depends on the porosity and the microstructure of the porous medium.

The inertial effect in periodic and random arrays has been the focus of a large number of studies. Koch and Ladd simulated moderate Reynolds number flows through periodic and random arrays of aligned cylinders [4] and spheres [5] in two and three dimensions. Their study showed that the inertial term made the transition from linear to quadratic in the random arrays. The inertial effect became smaller when the volume fraction approached close packing.

Direct numerical simulation appeared recently as a practical alternative to examine the transition of flow from linear to nonlinear behavior. For example, by solving the Navier-Stokes (NS) equations for a two-dimensional disordered structure with high porosity, Andrade et al. [6] demonstrated that the incipient departure from the Darcy law can be observed in the laminar regime of fluid flow without including turbulence effects. To date, mainly empirical relations have been used, such as the Ergun [7] and its components, the Carman-Kozeny (Blake-Kozeny) and Burke-Plummet equations, (Bird et al., [8]). Liu et al [9] devised a semi-empirical formula for the pressure gradient which incorporates the tortuosity, the curvature ratio and the variation of the pore cross-sectional area. Jackson and James [10] conducted a comprehensive review of the literature on a variety of theoretical models and presented a large collection of experimental data for both natural and synthetic fibrous media. Several authors also tried to derive these empirical laws by averaging the pore scale flow equations either using the homogenization technique [11] or the volume averaging method [12]. Many of these semi-empirical relations are only valid for a certain range of porosities and $\mathrm{Re}$ numbers. This has motivated the research towards the development of relationships to describe macroscopic parameters, such as permeability and the Darcy-Forchheimer term i.e. inertia term, for different kinds of porous media and flow regimes [13-14].

The objective of this paper is to present a more general form of the inertial term based on a detailed finite element (FE) simulation of a unidirectional steady fluid flow across a periodic array of two-dimensional cylinders at different porosity. Non-Darcy behavior is expected to play an important role due to the combination of high porosity and high pressure gradient. Comparisons are made to the Ergun and Forchheimer relations to examine their validity in this porosity and $\mathrm{Re}$ regime.

\section{MODEL DEVELOPMENT}

Previous studies of fluid flow in suspensions and fixed beds have been based on methods in which the velocity field is expressed in terms of multipole expansions [4]. These methods are suitable for Stokes flow [15] or inviscid flows [16]. To simulate the incompressible Navier-Stokes (NS) equations at finite values of the Reynolds number, we will use the finite element method (FEM). Both hexagonal and square arrays of parallel cylinders perpendicular to the flow direction are considered in this section as shown in Figure 1. Discretization is done, using unstructured triangular elements with $M$ grid points in the range of $12000<M<45000$

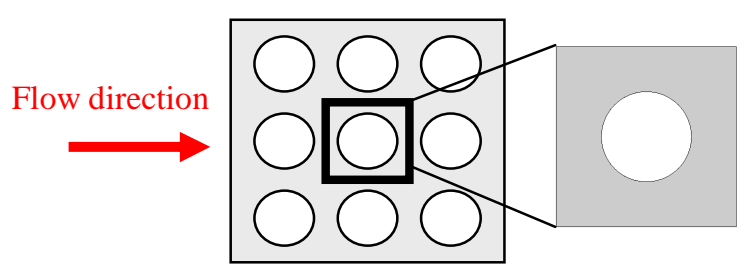

(a)

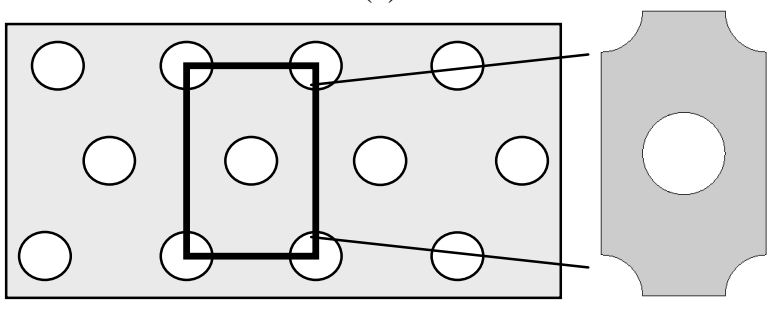

(b)

Fig. 1: The geometry of the unit cells used for (a) square and (b) hexagonal configurations, where the red arrow indicates the flow direction.

The basis of this model is the assumption that the porous media can be divided into representative volume elements (RVE) or unit cells. The particle friction factor is then determined by modelling the flow through these, more or less, idealized cells. At the left and right pressure- and at the top and bottom periodic-boundary conditions are applied. No-slip boundary condition, i.e., zero velocity is applied on the surface of the particles/fibers. The average/superficial velocity within the porous media in the unit cell is then defined as:

$U=\frac{1}{V} \int_{V_{f}} u d v=\varepsilon\langle u\rangle$

where $\varepsilon, u,\langle u\rangle, V$ and $V_{f}$ are porosity, the local microscopic velocity of the fluid, corresponding intrinsic phase averaged velocity, total volume of the unit cell and volume of the fluid, respectively. The superficial velocity is then calculated from the results of our FEM simulations. Knowing the viscosity $\mu$, the pressure drop $\Delta p$ over the length of the unit cell $L$, and using Eq. (1), the permeability $k$ is obtained. Simulating, only the unit 
cell/representative volume element (RVE) allows studying many different configurations and geometries. Nevertheless, some studies with larger systems were performed and showed that flow behaviour is independent of the system size for the periodic structures. This was confirmed not only for low Re, but also for $\operatorname{Re} \approx 1$.

\section{NUMERICAL RESULTS}

In this section some of the main results obtained through many high resolution FEM simulations are presented. In Figure 2 , the surface plot of the horizontal velocity field at constant pressure gradient $\Delta p / L=30[\mathrm{~Pa} / \mathrm{m}]$ is shown for the square configuration and different porosities. We define the particle Reynolds number $\operatorname{Re}_{p}$ as the ratio of quadratic inertia term to the Darcy viscous drag term:

$$
\operatorname{Re}_{p}=\frac{\rho U d_{p}}{(1-\varepsilon) \mu}
$$

where $d_{p}$ is the diameter of the particle/cylinder. The associated particle Reynolds number, $\operatorname{Re}_{p}$, for each porosity is also shown in Figure 2. In our simulations, the $\operatorname{Re}_{p}$ is varying between $1<\operatorname{Re}_{p}<100$
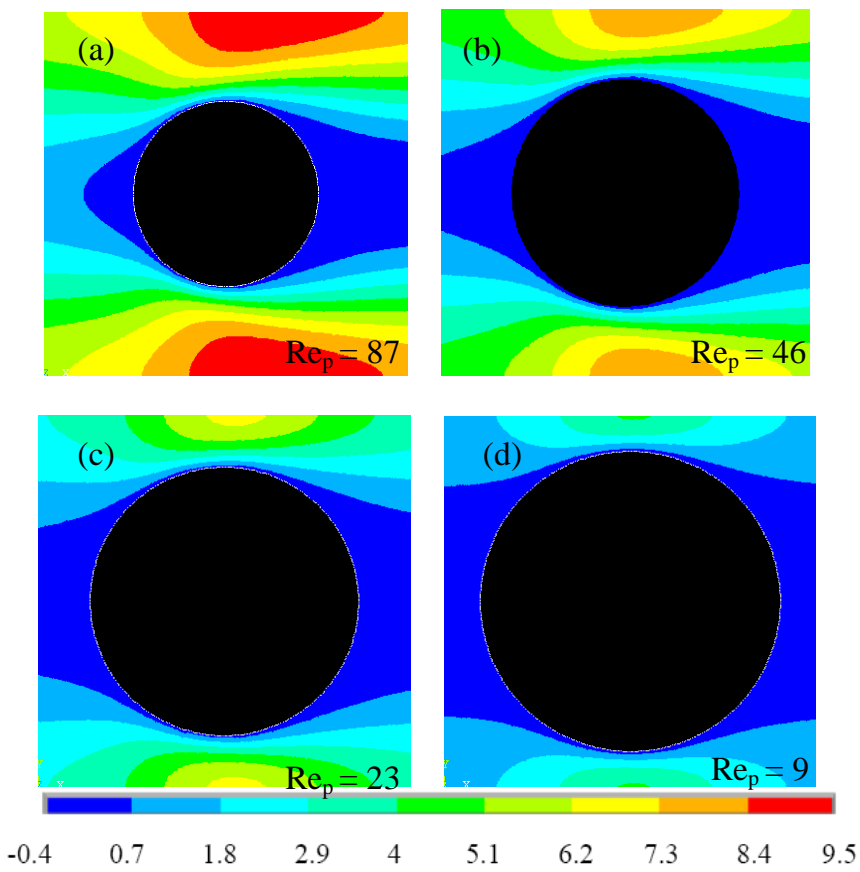

Fig. 2: Surface plot of the horizontal velocity field at constant pressure gradient $(\Delta p / L=30[\mathrm{~Pa} / \mathrm{m}])$ at different porosities (a) $\varepsilon=0.8$, (b) $\varepsilon=0.7$, (c) $\varepsilon=0.6$, (d) $\varepsilon=0.5$ for the square configuration.
It can be seen that at low porosities (Darcy's regime) we have the same velocity profile at inlet and outlet. This simply happens, because of mass balance, the symmetry of this geometry and no non-linearity in the flow. However, by increasing the porosity, the flow regime changes from Darcy to inertia (non-linear) and becomes non-symmetric with respect to the vertical axis.

We generalize Eq. (2) and assume that the pressure drop is related to the superficial velocity through the Darcy term, i.e., $\mu U / k$, plus an inertia term, which is a function of the fluid properties, porosity, particle diameter and superficial velocity $g\left(\varepsilon, \rho, \mu, d_{p}, U\right)$. Therefore we have:

$-\frac{\Delta p}{L}=\frac{\mu}{k} U+g\left(\varepsilon, \rho, \mu, d_{p}, U\right)$

In analogy to the definition of the Reynolds number, i.e. ratio of the nonlinear to the linear term in Eq. (5), we define a generalized Reynolds number $\mathrm{Re}$ as:

$\operatorname{Re}^{\prime}=\frac{g\left(\varepsilon, \rho, \mu, d_{p}, U\right)}{\mu U / k}$

and similarly a generalized/modified friction factor $f$, as the ratio of total drag to the inertial drag:

$f^{\prime}=-\frac{\Delta p / L}{g\left(\varepsilon, \rho, \mu, d_{p}, U\right)}$

Rewriting Eq. (5) in terms of the modified/generalized Reynolds number and friction factor we get:

$$
f^{\prime}=\frac{1}{\operatorname{Re}^{\prime}}+1
$$

In Figure 3 the variation of the modified friction factor versus modified Reynolds number is shown at different porosities for square configurations. The results collapse on the universal power law, i.e. Eq. (8).

Assuming that $f^{\prime}=10$ (the inertial drag became $10 \%$ of the total drag) is the limit of the non-Darcy effect [17], the critical $\operatorname{Re}$ is obtained as $\operatorname{Re}_{\mathrm{c}} \approx 0.1$ at which the modified friction factor starts to deviate from a straight line (see Figure 3). At sufficiently low $\mathrm{Re}^{\prime}$, the $1 / \mathrm{Re}$ term is dominant and all data collapse on the laminar regime (straight line). However, at $\operatorname{Re}^{\prime}>\operatorname{Re}_{\mathrm{c}}{ }_{\mathrm{c}}$, the second term becomes important and we can not neglect the inertia. Therefore, the data fits to Eq. (8). In order to be able to compare our results with previous results we define the particle friction factor $f_{p}$ as: 
$f_{p}=\frac{\nabla p d_{p}}{\rho U^{2}}$

The comparison of our square configuration FEM results with previous data at different porosities is shown in Figure 4. For completeness, the closed form relations of previous theories are listed in Table 1.

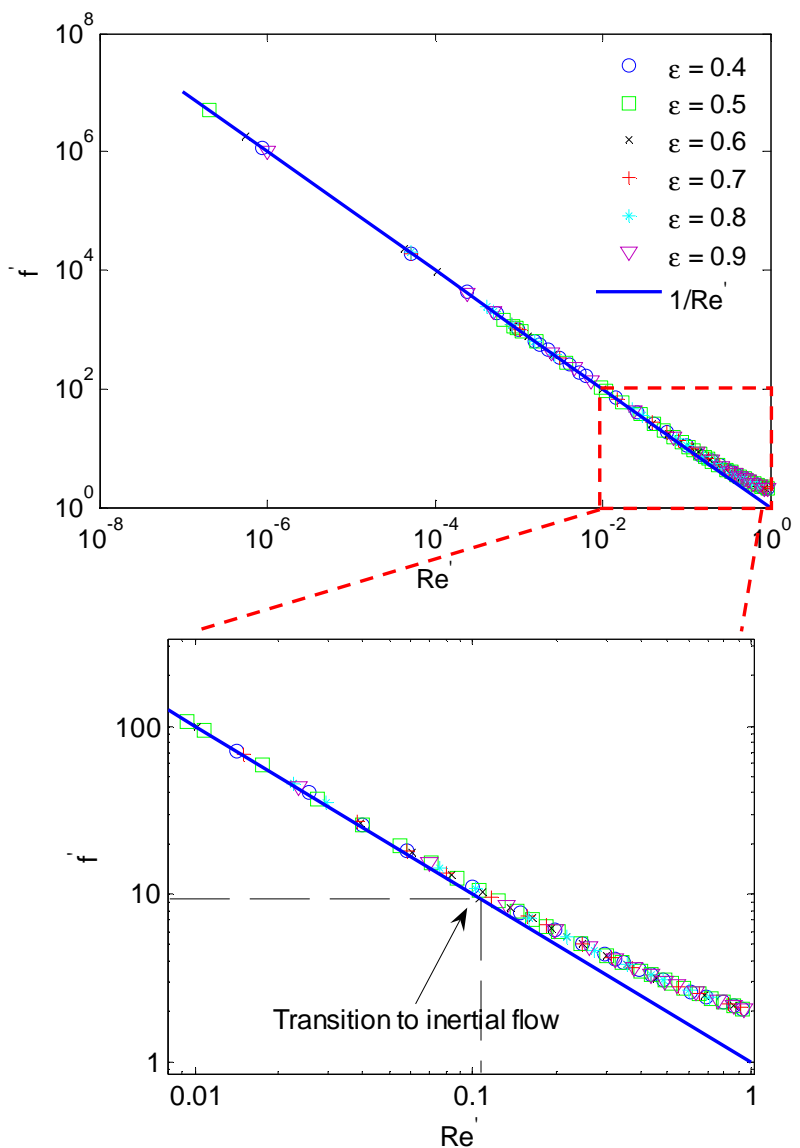

Fig. 3: Variation of the modified friction factor, $f$, versus modified Reynolds number, Re', at different porosities for square configurations. Transition to inertial regime occurs at

$$
\operatorname{Re}_{\mathrm{c}}{ }^{\prime} \approx 0.1
$$

It is seen that at low porosities and high $\mathrm{Re}_{p}$, our results approach results of Kurten et al. [19] but at low $\mathrm{Re}_{p}$, the results of Benyahia et al. [18] are closer. It should be noted that previous results, namely, Benyahia et al. [18] and Kurten et al. [19] were the modified Forchheimer equations for granular packed beds, i.e. 3D random spherical particle packing. However, our results are for regular two-dimensional square configurations. To our best of knowledge, there does not exist a 2-D relation in this range of $\mathrm{Re}_{p}$ and it is just an attempt to see if the 3-D relations could be also relevant for 2-D cylinders.
The relation between the proposed modified Reynolds number, Re', and the particle Reynolds number, $\mathrm{Re}_{p}$, is shown in Figure 5. As we see, this relation also depends on the pore structure, namely square or hexagonal configuration, and on the porosity.

Table1: Available modifications of Ergun's equation [7], namely, Benyahia et al. [18] and Kurten et al. [19], in terms of particle friction factor $f_{p}$, and particle Reynolds number $\operatorname{Re}_{p}$

\begin{tabular}{|c|c|c|}
\hline Ref. & $f_{p}$ & $\begin{array}{c}\text { Range of } \\
\text { validity }\end{array}$ \\
\hline$[7]$ & $\left(\frac{1-\varepsilon}{\varepsilon^{3}}\right)\left(\frac{150}{\operatorname{Re}_{p}}+1.75\right)$ & $\varepsilon<0.8$ \\
\hline$[18]$ & $\frac{180(1-\varepsilon)}{\operatorname{Re}_{p} \varepsilon^{3}}+9 F_{3}(1-\varepsilon)$ & $\varepsilon<0.6$, \\
& $\begin{array}{c}F_{3}=0.0673+0.212(1-\varepsilon)+0.0232 \varepsilon^{-5} \\
F_{1}=0.11+0.00051 e^{11.6(1-\varepsilon)}\end{array}$ & $\operatorname{Re}_{p}>\frac{2 F_{3}}{F_{1}(1-\varepsilon)}$ \\
\hline$[19]$ & $\left(\frac{25}{4 \varepsilon^{3}}(1-\varepsilon)^{2}\right)\left(21 \operatorname{Re}_{p}^{-1}+6 \operatorname{Re}_{p}^{-0.5}+0.28\right)$ & $0.1<\operatorname{Re}_{\mathrm{p}}<4000$ \\
\hline
\end{tabular}

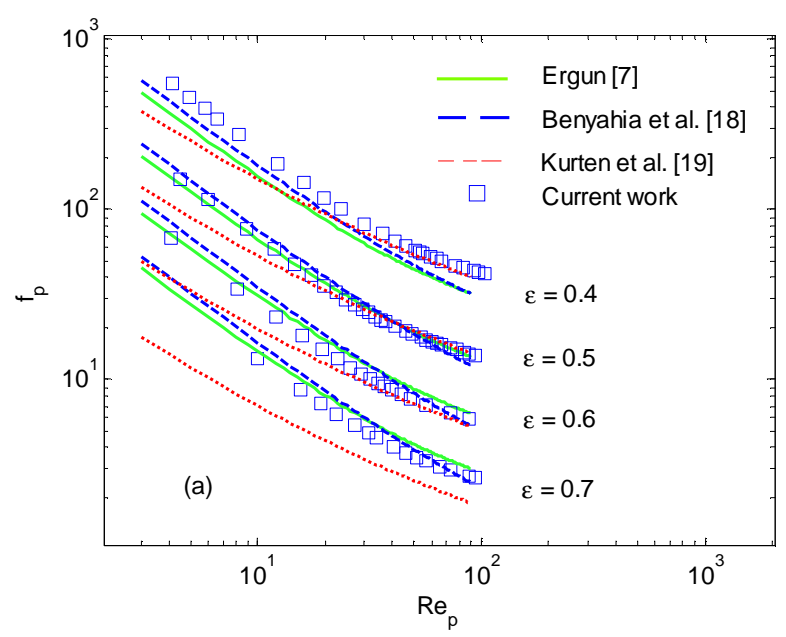

Fig. 4: Variation of particle friction factor $f_{p}$ versus particle Reynolds number, $\operatorname{Re}_{p}$ at different porosities for square configuration

At the same modified Reynolds number Re', a hexagonal configuration has a higher $\operatorname{Re}_{p}$ compared to a square geometry. This geometry effect can be neglected at creeping flow (sufficiently low $\mathrm{Re}_{p}$ ). 


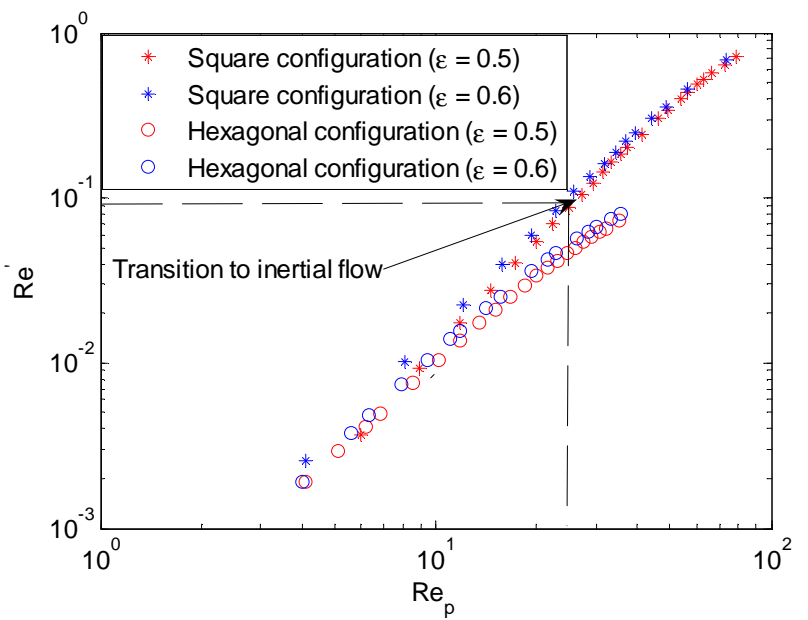

Fig. 5: Variation of the modified Reynolds number, Re', versus particle Reynolds number, $\operatorname{Re}_{p}$, for different porosities and configurations

The inertial drag term $g$ in Eq. (5) can be directly obtained from our numerical simulations $(g=-\Delta p / L-\mu U / k)$. In Figure 6 we plot $g$ as a function of average velocity. It is observed that for all range of porosities studied here, the non-Darcy (inertia) flow scales badly with a quadratic law, for both square and hexagonal configurations (hexagonal results are not shown). However, at low porosities, the power approaches to three, i.e., a cubic law. An even better fit can be obtained when the inertia term is expressed as:

$g\left(\varepsilon, \rho, \mu, d_{p}, U\right)=C_{1} U^{C_{2}}$

where the constants $C_{1}$ and $C_{2}$ depend on the porosity $\varepsilon$, diameter of particles/cylinders $d_{p}$ and the fluid properties i.e. $\mu$ and $\rho$. The values of $C_{1}$ and $C_{2}$ are listed in Table 2. By applying a simple dimensional analysis, the non-dimensional form of Eq. (10) is written as:

$g\left(\varepsilon, \rho, \mu, d_{p}, U\right)=\frac{\mu^{2}}{\rho d_{p}^{3}} C_{1}^{\prime}\left(\frac{U}{U^{*}}\right)^{C_{2}^{\prime}}$

with $U^{*}=\mu /\left(\rho d_{p}\right)$, where $C_{1}^{\prime}, C_{2}^{\prime}$ are now dimensionless functions of porosity. Basically, the values of $C_{2}$ ' are the same as $C_{2}$, and the values of $C_{1}$ ' are listed in Table 2. As we see, the power, $C_{2}$ ', is not exactly 2 or 3 but, depending on the porosity, varies between these two values. These numerical results show that the prefactor, $C_{1}$, is sensitive to the pore structure, but also the power, $C_{2}$ ' has some dependence.

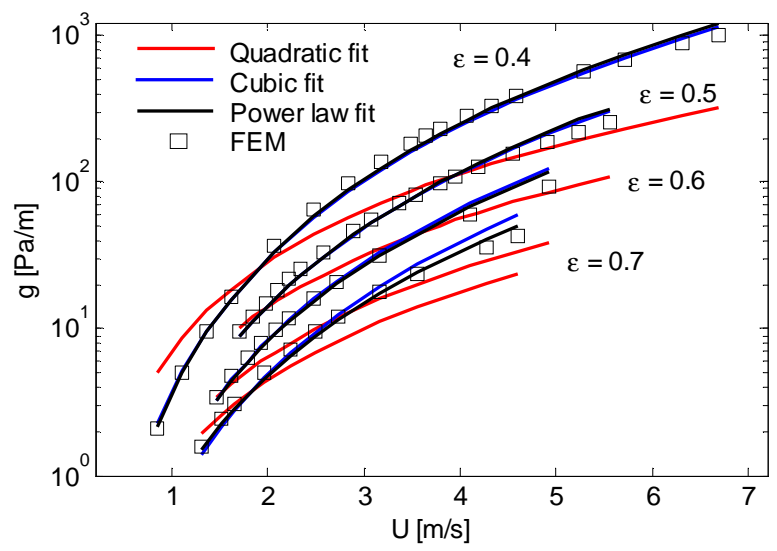

Fig. 6: Variation of non-Darcy drag term, $g$, as a function of superficial/average velocity at different porosities for the square configuration

Table 2: The porosity dependent parameters, $C_{1}, C_{1}$ ' and $C_{2}$, of the inertial term, $g$, for different porosities and configurations

\begin{tabular}{|c|c|c|c|}
\hline \multicolumn{4}{|c|}{ Square configuration } \\
\hline Porosity $(\varepsilon)$ & $C_{1}$ & $C_{2}=C_{2}{ }^{\prime}$ & $C_{1}^{\prime}$ \\
\hline 0.9 & 0.27875 & 2.57306 & 0.04798 \\
\hline 0.85 & 0.36178 & 2.70182 & 0.05616 \\
\hline 0.8 & 0.45309 & 2.75827 & 0.067 \\
\hline 0.75 & 0.54514 & 2.81237 & 0.07542 \\
\hline 0.7 & 0.68878 & 2.8157 & 0.09635 \\
\hline 0.65 & 0.87913 & 2.87533 & 0.11139 \\
\hline 0.6 & 1.08037 & 2.93689 & 0.1223 \\
\hline 0.55 & 1.40838 & 2.95679 & 0.15371 \\
\hline 0.5 & 1.68504 & 3.03325 & 0.16389 \\
\hline 0.45 & 2.59037 & 3.02521 & 0.24553 \\
\hline 0.4 & 3.69622 & 3.04657 & 0.33413 \\
\hline & Hexagonal configuration & \\
\hline 0.9 & 0.222299 & 2.6113 & 0.03546 \\
\hline 0.85 & 0.255741 & 2.5963 & 0.045081 \\
\hline 0.8 & 0.293749 & 2.6265 & 0.052344 \\
\hline 0.75 & 0.343661 & 2.6142 & 0.065173 \\
\hline 0.7 & 0.385963 & 2.6525 & 0.070941 \\
\hline 0.65 & 0.460649 & 2.663 & 0.085267 \\
\hline 0.6 & 0.542535 & 2.6785 & 0.099765 \\
\hline 0.55 & 0.671844 & 2.6919 & 0.12261 \\
\hline 0.5 & 0.830711 & 2.7073 & 0.149445 \\
\hline 0.45 & 1.086687 & 2.7283 & 0.189903 \\
\hline 0.4 & 1.445991 & 2.7343 & 0.252344 \\
\hline & & & \\
\hline & & & \\
\hline & & &
\end{tabular}




\section{CONCLUDING REMARKS}

Based on FEM simulations, we analyzed the transverse flow through unidirectional, regular, two dimensional fibrous media in both laminar and inertial regimes. By increasing the pressure gradient, we observed a typical departure from Darcy's law (creeping flow) at sufficiently high Reynolds numbers, Re. Some conclusions can be drawn as follows:

- The correlation between particle friction factor, $f_{p}$, and particle Reynolds number, $\operatorname{Re}_{p}$, obtained from our numerical simulations, are compared with available theoretical predictions like the Ergun equation. It is observed that the particle friction factor is not only a function of porosity and $\mathrm{Re}_{\mathrm{p}}$, but also depends on the pore structure (see Figure 4).

- All relations are just valid in a certain porosity and $\mathrm{Re}_{p}$ range and also pore structure in 2-D, i.e. square or hexagonal configurations.

- Both quadratic and cubic laws, which are often used as extensions of the Darcy law for inertial flows through porous media, are reformulated in a more general non-dimensional power law form (Eq. (11)). The numerical results show that the power law model can fit the numerical results better than integer power 2 or 3 in the range of $U$ studied here.

- By defining the modified Reynolds number, Re', and friction factor, $f$ ', a universal relation between these two key parameters is introduced. It is valid for square and hexagonal configurations in a broad range of porosities (Eq. (8)). The transition (based on $10 \%$ inertia) from creeping to inertial flow occurs at $\operatorname{Re}_{\mathrm{c}}{ }^{\prime} \approx 0.1$ which is equivalent to $\operatorname{Re}_{p} \approx 25$.

Future work will investigate random arrangements of cylinders and the effects of boundary conditions and size of the RVE on the permeability. These results could be utilized for verification and validation of advanced models for particle fluid interaction and for the coupling of the discrete element method (DEM) with FEM or CFD in a multiscale coarse grid mesh.

\section{ACKNOWLEDGMENTS}

The authors would like to thank N.P. Kruyt for helpful discussion and the financial support of STW through the STWMuST program, project number 10120.

\section{REFERENCES}

[1] Deen N.G., Van Sint Annaland M., Van der Hoef M.A., Kuipers J.A.M., Chemical Engineering Science; 62 (2007) $28-44$.
[2] Tsuji Y., Kawaguchi T., Tanaka T., Powder Technol.; 77 (1993) 79-87.

[3] Forchheimer P., Wasserbewegung durch Boden, Z. Ver. Deutsch. Ing.; 45 (1901), 1782.

[4] Koch, D. and Ladd, A. J. C., J. Fluid Mech.; 239 (1997), 31-66.

[5] Hill, R. J., Koch, D. L., and Ladd, A. J. C., J. Fluid Mech.; 448 (2001), 243-278.

[6] Andrade, J. A. Jr., Costa, U. M. S., Almeida, M. P., Makse, H. A. and Stanley, H. E., Phys. Rev. Lett.; 82 (1998), 5249-5252.

[7] Ergun, S., Chem. Eng. Prog.; 48 (1952), 89-94.

[8] Bird RB, Stewart WE, Lightfoot EN (2002) Transport phenomena, 2nd edn. Wiley, New York.

[9] Liu S, Afacan A and Masliyah J, Chem. Eng. Sci.; 49 (1994), 3565-86.

[10] Jackson GW, James DF. Can J Chem Eng; 64 (1986); 364-74.

[11] Sanchez-Palencia E., Lecture Notes in Physics. Springer; 127 (Springer Verlag, Berlin, 1980).

[12] Whitaker S., Transport Porous Media; 1 (1986); 3-25.

[13] Papathanasiou T.D., Markicevic B., Phys. Fluids; 13 (2001), 2795-2804.

[14] D.G. Roychowdhury, S.K. Das, T. Sundararajan, Int. J. Numer. Methods Fluids; 39 (2002), 23-40.

[15] Brady, J. F., Bossis, G., Ann. Rev. Fluid Mech.; 20 (1988), 111.

[16] Sangani, A. S., Didwania, A. K., J. Fluid mech.; 250 (1993), 307.

[17]Zeng Z. and Grigg R., Transport in Porous Media; 63 (2006), 57-69.

[18]Benyahia S., Syamlal M., and O'Brien T.J., Powder Technology; 162 (2006), 166-174.

[19] Kürten H., Raasch J. and Rumpf H., B, Chem. Ing. Tech.; 38 (1966), 941-948. 